\title{
Kaon condensation and composition of neutron star matter in modified quark-meson coupling model
}

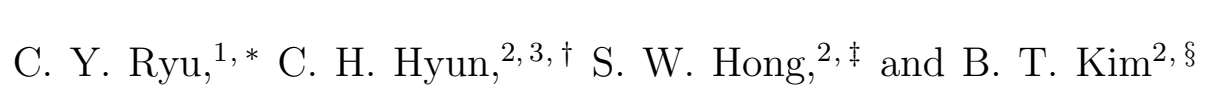 \\ ${ }^{1}$ Department of Physics and Basic Atomic Energy Research Institute, \\ Sungkyunkwan University, Suwon 440-746, Korea \\ ${ }^{2}$ Department of Physics and Institute of Basic Science, \\ Sungkyunkwan University, Suwon 440-746, Korea \\ ${ }^{3}$ School of Physics, Seoul National University, Seoul 151-742, Korea
}

(Dated: March 9, 2007)

\begin{abstract}
We use the modified quark-meson coupling (MQMC) model to study the composition profile of neutron star matter and compare the results with those calculated by quantum hadrodynamics (QHD). Both MQMC and QHD model parameters are adjusted to produce exactly the same saturation properties so that we can investigate the model dependences of the matter composition at high densities. We consider the possibility of deep kaon optical potential and find that the composition of matter is very sensitive to the interaction strength of kaons with matter. The onset densities of the kaon condensation are studied in detail by varying the kaon optical potentials. We find that the MQMC model produces the kaon condensation at lower densities than QHD. The presence of kaon condensation changes drastically the population of octet baryons and leptons. Once the kaon condensation takes place, the population of kaons builds up very quickly, and kaons become the dominant component of the matter. We find that the $\omega$-meson plays an important role in increasing the kaon population and suppressing the hyperon population.
\end{abstract}

\footnotetext{
*Electronic address: cyryu@rcnp.osaka-u.ac.jp

${ }^{\dagger}$ Electronic address: hch@meson.skku.ac.kr

${ }^{\ddagger}$ Electronic address: swhong@skku.ac.kr

$\S$ Electronic address: btkim@skku.ac.kr
} 


\section{INTRODUCTION}

Observation of neutron star properties such as mass, size and temperature provides us with important clues to the understanding of the state of matter at extremely high densities. In the 1970's, the maximum mass of the neutron star was calculated with the $N N$ potentials available at that time [1, 2, 3, 4] and mean field models [5, 6]. Most of the calculations done in the 1970's resulted in stiff equations of state, and thus the maximum mass of a neutron star was predicted to be larger than $2 M_{\odot}$, where $M_{\odot}$ is the solar mass. Only the Reid soft core potential yielded a soft equation of state and consequently a small maximum mass of a neutron star, $1.6 M_{\odot}$ [1]. Recent observations of the masses of binary pulsars [7], which are candidates of neutron stars indicate that the maximum mass of neutron stars are roughly around $1.5 M_{\odot}$, substantially smaller than most of the predicted values in the 1970's. (However, very recent observations seem to suggest possible existence of more massive pulsars in the range $(1.8-2.0) M_{\odot}[8,9]$, though further confirmation is needed.) On the other hand, exotic forms of matter, i.e., matter consisting of degrees of freedom other than the nucleons have been proposed already many years ago. Some of the proposed exotic states of matter include those with creation of hyperons [10], Bose-Einstein condensation (pions [11] or kaons [12]), strange matter [13], and quark deconfinement [14, 15, 16]. These exotic states seem to reduce the maximum mass of a neutron star close to the observations [17, 18, 19], implying that exotic degrees of freedom seem to be needed to reproduce the observed masses of neutron stars.

In this work, we consider the strangeness degrees of freedom by including both hyperon creation and kaon condensation in the neutron star matter. (It is the anti-kaon that matters here, but we simply refer to both kaons and anti-kaons as kaons for brevity.) The masses and energies of the hyperons and kaons in medium are sensitive to their interactions with the surrounding matter. In the meson-exchange picture, meson-hyperon and meson-kaon coupling constants can fix the strength of these interactions. The meson-hyperon coupling constants may be determined from the binding energies of hyperons in hypernuclei. The meson-kaon coupling constants have been studied by using the kaon-nucleon scattering [20, 21] and kaonic atom data [20]. Recently, the magnitudes of the kaon-nucleus potential in matter have attracted much attention. Some calculations [20, 22, 23] show that the real part of the $K^{-}$-nucleus optical potential $U_{K^{-}}$is shallow $\left(U_{K^{-}} \approx-50 \mathrm{MeV}\right)$, but some other 
calculations suggest that $U_{K^{-}}$can be as large as about $-120 \mathrm{MeV}[21,24]$ or even close to $-200 \mathrm{MeV}[25]$.

Akaishi and Yamazaki predicted possible existence of deeply bound kaonic nuclei [26], in which $U_{K^{-}}$at normal density $\rho_{0}$ was estimated to be about $-120 \mathrm{MeV}$. Then, experiments at KEK claimed the observation of tribaryon kaonic nuclei, $\mathrm{S}^{0}$ [27] and $\mathrm{S}^{+}$[28], which seem to suggest that $K^{-}$may be even more deeply bound than the theoretical prediction [26] (The former claim [27], however, was withdrawn by the experimental group [29]). FINUDA collaboration at DA $\Phi$ NE [30] and a BNL experiment with ${ }^{16} \mathrm{O}\left(K^{-}, n\right)$ reaction [31] also reported distinct peaks. More recently there was a theoretical work which considered large kaonic binding energies and calculated widths of kaonic nuclear bound states [32]. The identities of these experimental peaks need to be studied further experimentally and theoretically. However, in this work, we consider the possibility of deep optical potential of kaons in nuclei and explore the consequences in the composition profile of neutron star matter.

In this work, for the description of dense matter we employ the modified quark-meson coupling (MQMC) model [33]. Nucleons and hyperons in the baryon octet are treated as MIT bags. The bag constant $B_{B}$ and phenomenological constant $Z_{B}$ for a baryon $B$ are fixed to reproduce the free mass of each baryon $B$. Coupling constants between $(u, d)$ quarks in the bags and $(\sigma, \omega, \rho)$-mesons are adjusted to give us the binding energy per a nucleon $E_{b} / A=16 \mathrm{MeV}$ and symmetry energy $a_{\text {sym }}=32.5 \mathrm{MeV}$ at the saturation density $\rho_{0}=0.17 \mathrm{fm}^{-3}$. Since the interaction between the $s$-quark and mesons are not well known, we adopt the standard quark counting rule and assume the $s$-quark is decoupled from $(\sigma$, $\omega, \rho)$-mesons. To take into account the interactions between $s$-quarks, we introduce $\sigma^{*}(980)$ and $\phi(1020)$ mesons following Ref. [18] for the baryon and Ref. [34] for the kaon. We also assume the kaon as a point particle. This treatment allows us to use $U_{K^{-}}$as an input to fix the coupling constant between the $\sigma$-meson and the kaon, $g_{\sigma K}$. In our model the real part of the kaon optical potential at $\rho=\rho_{0}$ can be written as $U_{K^{-}}=-\left(g_{\sigma K} \sigma\left(\rho_{0}\right)+g_{\omega K} \omega\left(\rho_{0}\right)\right)$, where $\sigma\left(\rho_{0}\right)$ and $\omega\left(\rho_{0}\right)$ are the values of the meson fields at $\rho_{0}$. Using the value of $g_{\omega K}$ given by the quark counting rule, we can fix $g_{\sigma K}$ for each given value of $U_{K^{-}}$. Once the parameters of the model are fixed, the composition profile of neutron star matter can be obtained from the $\beta$-equilibrium and charge neutrality. We find that the composition of neutron star matter changes dramatically depending on the value of $U_{K^{-}}$.

To investigate the model dependence of the results we also employ quantum hadrody- 
namics (QHD) model [35] for calculating the composition of matter. The parameters of the QHD model are calibrated to produce exactly the same saturation properties as in the MQMC model. Our calculations show that the onset densities of the kaon condensation and the compositions of matter at high densities are substantially model dependent. In Sect. II, we introduce model Lagrangians and fix the model parameters. The results are discussed in Sect. III. Conclusions and discussions follow in Sect. IV.

\section{THEORY}

In this section we first briefly sketch the MQMC and QHD models by presenting the model Lagrangians. The models are calibrated so as to be consistent with each other at the saturation density by fixing the coupling constants of both models to produce exactly the same saturation properties; the saturation density, the binding energy, the symmetry energy, the nucleon effective mass, and the compression modulus. We then show how the physical quantities that will determine the composition of the neutron star matter can be obtained self-consistently.

\section{A. Models}

The model Lagrangian comprises the terms for the octet baryons, exchange mesons, leptons and kaons; $\mathcal{L}_{t o t}=\mathcal{L}_{B}+\mathcal{L}_{M}+\mathcal{L}_{l}+\mathcal{L}_{K}$. Octet baryon, exchange meson and lepton terms in the mean field approximation can be written as

$$
\begin{aligned}
\mathcal{L}_{B} & =\sum_{B} \bar{\psi}_{B}\left[i \gamma \cdot \partial-m_{B}^{*}\left(\sigma, \sigma^{*}\right)-\gamma^{0}\left(g_{\omega B} \omega_{0}+g_{\phi B} \phi_{0}+\frac{1}{2} g_{\rho B} \tau_{z} \rho_{03}\right)\right] \psi_{B} \\
\mathcal{L}_{M} & =-\frac{1}{2} m_{\sigma}^{2} \sigma^{2}-\frac{1}{2} m_{\sigma^{*}}^{2} \sigma^{* 2}+\frac{1}{2} m_{\omega}^{2} \omega_{0}^{2}+\frac{1}{2} m_{\phi}^{2} \phi_{0}^{2}+\frac{1}{2} m_{\rho}^{2} \rho_{03}^{2}, \\
\mathcal{L}_{l} & =\sum_{l} \bar{\psi}_{l}\left(i \gamma \cdot \partial-m_{l}\right) \psi_{l},
\end{aligned}
$$

where $B$ denotes the sum over all the octet baryons $\left(p, n, \Lambda, \Sigma^{+}, \Sigma^{0}, \Sigma^{-}, \Xi^{0}, \Xi^{-}\right)$, and $l$ stands for the sum over the free electrons and muons $\left(e^{-}, \mu^{-}\right) . \sigma, \omega$ and $\rho$ mesons mediate the interactions between the non-strange light quarks $(u$ and $d)$. $\sigma^{*}$ and $\phi$ mesons are introduced to take into account the interactions between $s$ quarks. $\mathcal{L}_{B}$ is of the identical form for both the MQMC and QHD models, but differs in the definition of the effective baryon mass $m_{B}^{*}$ as will be shown below. 


\section{MQMC}

In the MQMC model, a baryon is a composite system with quarks in a spherical bag, and its mass is given in terms of bag parameters and quark eigenenergy. The effective mass of a baryon in matter $m_{B}^{*}\left(\sigma, \sigma^{*}\right)$ can be written as [18, 33, 36, 37, 38]

$$
m_{B}^{*}=\sqrt{E_{B}^{2}-\sum_{q}\left(\frac{x_{q}}{R}\right)^{2}} .
$$

The bag energy of a baryon is given by

$$
E_{B}=\sum_{q} \frac{\Omega_{q}}{R}-\frac{Z_{B}}{R}+\frac{4}{3} \pi R^{3} B_{B}
$$

where $B_{B}$ and $Z_{B}$ are the bag constant and a phenomenological constant for the zero-point

motion of a baryon $B$, respectively. $\Omega_{q}=\sqrt{x_{q}^{2}+\left(R m_{q}^{*}\right)^{2}}$, where $m_{q}^{*}\left(=m_{q}-g_{\sigma}^{q} \sigma-g_{\sigma^{*}}^{q} \sigma^{*}\right)$ is the effective mass of a quark whose free mass is $m_{q}$. We take $m_{q}=0$ for $q=u, d$ and $m_{q}=150 \mathrm{MeV}$ for $q=s$. (Other choices of $m_{q=s}$ values do not make differences in the results [39].) $x_{q}$ is determined from the boundary condition on the bag surface $r=R$,

$$
j_{0}\left(x_{q}\right)=\beta_{q} j_{1}\left(x_{q}\right),
$$

where $\beta_{q}=\sqrt{\frac{\Omega_{q}-R m_{q}^{*}}{\Omega_{q}+R m_{q}^{*}}}$. In the MQMC model, the bag constant $B_{B}$ is assumed to depend on density [33, 38]. In this work, we use the extended form in Ref. [18] to include the contribution from $\sigma^{*}$ as

$$
B_{B}\left(\sigma, \sigma^{*}\right)=B_{B 0} \exp \left\{-\frac{4}{m_{B}}\left(g_{\sigma}^{\prime B} \sum_{q=u, d} n_{q} \sigma+g_{\sigma^{*}}^{\prime B}\left(3-\sum_{q=u, d} n_{q}\right) \sigma^{*}\right)\right\},
$$

where $m_{B}$ is the free mass of the baryon $B$, assuming that the $\sigma$ meson couples to $u$ and $d$ quarks only and that the $\sigma^{*}$ meson couples to the $s$ quark only.

\section{QHD}

In the QHD model, a baryon is treated as a point particle, and thus its effective mass is simply written as

$$
m_{B}^{*}=m_{B}-g_{\sigma B} \sigma-g_{\sigma^{*} B} \sigma^{*} .
$$

In order to reproduce the same saturation properties as obtained in the MQMC model, self-interactions of the $\sigma$-field [40]

$$
U_{\sigma}^{\mathrm{QHD}}=\frac{1}{3} g_{2} \sigma^{3}+\frac{1}{4} g_{3} \sigma^{4}
$$


are added to Eq. (2) so that

$$
\mathcal{L}_{M}^{\mathrm{QHD}}=\mathcal{L}_{M}-U_{\sigma}^{\mathrm{QHD}}
$$

As mentioned above, the baryon and the lepton Lagrangians for the QHD model take the form given by Eqs. (11) and (3).

\section{Kaon}

The effective Lagrangian for the kaon may be expressed as [41]

$$
\mathcal{L}_{K}=D_{\mu}^{*} K^{*} D^{\mu} K-m_{K}^{*} K^{*} K
$$

where $D_{\mu}=\partial_{\mu}+i g_{\omega K} \omega_{\mu}-i g_{\phi K} \phi_{\mu}+i \frac{1}{2} g_{\rho K} \vec{\tau} \cdot \vec{\rho}_{\mu}$. In this work we treat the kaon as a point particle in both MQMC and QHD models, and its effective mass is given by

$$
m_{K}^{*}=m_{K}-g_{\sigma K} \sigma-g_{\sigma^{*} K} \sigma^{*}
$$

The equation of motion for a kaon is given by

$$
\left[D_{\mu} D^{\mu}+m_{K}^{* 2}\right] K(x)=0 .
$$

In uniform infinite matter, the kaon field $K(x)$ can be written as a plane wave. Substituting the plane wave solution into the equation of motion, we obtain the dispersion relation for the anti-kaon

$$
\omega_{K}=m_{K}^{*}-g_{\omega K} \omega_{0}+g_{\phi K} \phi_{0}-g_{\rho K} \frac{1}{2} \rho_{03} .
$$

\section{B. Model parameters}

\section{MQMC}

In the MQMC model, MIT bag parameters $B_{B 0}$ and $Z_{B}$ are determined to reproduce the free mass of a baryon $B,\left.m_{B}^{*}\right|_{\rho=0}=m_{B}$ with the minimization condition $\left.\frac{\partial m_{B}}{\partial R}\right|_{R=R_{0}}=0$ at a free bag radius $R_{0}$, which we choose as $R_{0}=0.6 \mathrm{fm}$. The bag parameters $B_{B 0}$ and $Z_{B}$ for the octet baryons are listed in Table I.

Three saturation conditions $\rho_{0}, E_{b} / A$, and $a_{\text {sym }}$ could determine three quark-meson coupling constants $g_{\sigma}^{u, d}, g_{\omega}^{u, d}$ and $g_{\rho}^{u, d}$, assuming $u$ and $d$ quarks to be identical in the isodoublet. The MQMC model, however, introduces an additional constant $g_{\sigma}^{\prime B}$ in Eq. (7). Thus we 


\begin{tabular}{|c|c|c|c|}
\hline$B$ & $m_{B}(\mathrm{MeV})$ & $B_{B 0}^{1 / 4}(\mathrm{MeV})$ & $Z_{B}$ \\
\hline$N$ & 939.0 & 188.1 & 2.030 \\
\hline$\Lambda$ & 1115.6 & 197.6 & 1.926 \\
\hline$\Sigma^{+}$ & 1189.4 & 202.7 & 1.829 \\
\hline$\Sigma^{0}$ & 1192.0 & 202.9 & 1.826 \\
\hline$\Sigma^{-}$ & 1197.3 & 203.3 & 1.819 \\
\hline$\Xi^{0}$ & 1314.7 & 207.6 & 1.775 \\
\hline$\Xi^{-}$ & 1321.3 & 208.0 & 1.765 \\
\hline
\end{tabular}

TABLE I: Bag constants $B_{B 0}$ and phenomenological constants $Z_{B}$ for octet baryons to reproduce the free mass of each baryon. The bag radius is chosen as $R_{0}=0.6 \mathrm{fm}$ for all octet baryons, and the bare masses of quarks are fixed as $m_{u(d)}=0 \mathrm{MeV}$ and $m_{s}=150 \mathrm{MeV}$.

\begin{tabular}{|c|c|c|c||c|c|}
\hline$g_{\sigma}^{q}$ & $g_{\omega}^{q}$ & $g_{\sigma}^{\prime B}$ & $g_{\rho}^{q}$ & $m_{N}^{*} / m_{N}$ & $K(\mathrm{MeV})$ \\
\hline 1.0 & 2.71 & 2.27 & 7.88 & 0.78 & 285.5 \\
\hline
\end{tabular}

TABLE II: The coupling constants between $(u, d)$-quarks and $(\sigma, \omega, \rho)$-mesons in the MQMC model to reproduce the binding energy $E_{b} / A=16 \mathrm{MeV}$ and symmetry energy $a_{\text {sym }}=32.5 \mathrm{MeV}$ at the saturation density $0.17 \mathrm{fm}^{-3} \cdot m_{N}^{*} / m_{N}$ and $K$ are the ratio of the effective mass to the free mass of the nucleon and the compression modulus at the saturation density, respectively.

fix $g_{\sigma}^{u, d}=1$, and adjust the remaining three constants to meet the three conditions. The resulting coupling constants are given in Table II together with the ratio of the effective mass of the nucleon $m_{N}^{*} / m_{N}$ and the compression modulus $K \cdot m_{N}^{*}$ and $K$ are within reasonable ranges: $m_{N}^{*}=(0.7 \sim 0.8) m_{N}$ and $K=(200 \sim 300) \mathrm{MeV}$.

The coupling constants between s-quarks and mesons cannot be determined from the saturation properties. In principle, experimental data from hypernuclei and kaon-nucleus scattering could be used to determine the coupling constants between $s$-quarks and mesons (for example, see Ref. [42]). However, these coupling constants are not well known yet, and for simplicity we assume that the quark counting rule holds and that the $s$ quark does not interact with $u$ and $d$ quarks. Then we have

$$
g_{\sigma}^{s}=g_{\omega}^{s}=g_{\rho}^{s}=g_{\sigma *}^{u, d}=g_{\phi}^{u, d}=0 .
$$




\begin{tabular}{|c|c|c|c|c|}
\hline$g_{\sigma N}$ & $g_{\omega N}$ & $g_{\rho N}$ & $g_{2}\left(\mathrm{fm}^{-1}\right)$ & $g_{3}$ \\
\hline 8.06 & 8.19 & 7.88 & 12.139 & 48.414 \\
\hline
\end{tabular}

TABLE III: The meson-nucleon coupling constants and the coefficients of the $\sigma$-meson self interaction terms used in the QHD model. They reproduce the same saturation properties as in the MQMC model; $\rho_{0}=0.17 \mathrm{fm}^{-3}, E_{b}=16 A \mathrm{MeV}, a_{\mathrm{sym}}=32.5 \mathrm{MeV}, m_{N}^{*}=0.78 m_{N}$ and $K=285.5$ $\mathrm{MeV}$.

To fix the meson-baryon coupling constants in the model Lagrangian, we also use the quark counting rule

$$
\begin{aligned}
\frac{1}{3} g_{\omega N} & =\frac{1}{2} g_{\omega \Lambda}=\frac{1}{2} g_{\omega \Sigma}=g_{\omega \Xi}=g_{\omega}^{q}, \\
g_{\rho N} & =g_{\rho \Sigma}=g_{\rho \Xi}=g_{\rho}^{q}, \quad g_{\rho \Lambda}=0 \\
g_{\phi \Lambda} & =g_{\phi \Sigma}=\frac{1}{2} g_{\phi \Xi}=g_{\phi}^{s},
\end{aligned}
$$

and the $\mathrm{SU}(6)$ symmetry

$$
\begin{aligned}
g_{\sigma *}^{s} & =\sqrt{2} g_{\sigma}^{u, d}=\sqrt{2}, \\
g_{\phi}^{s} & =\sqrt{2} g_{\omega}^{u, d}=3.83, \\
g_{\sigma^{*}}^{\prime B} & =\sqrt{2} g_{\sigma}^{\prime B} .
\end{aligned}
$$

The quark-meson coupling constants $g_{\omega}^{q}$ and $g_{\rho}^{q}$ given in Table II and the relations in Eqs. (15)-(17) determine all the meson-baryon coupling of the MQMC model.

\section{QHD}

In the QHD model, $g_{\sigma N}$ and $g_{\omega N}$ are adjusted to yield $\rho_{0}$ and $E_{b}$, and $g_{\rho N}$ is fitted to produce $a_{\text {sym. }} \cdot g_{2}$ and $g_{3}$ in $U_{\sigma}^{\mathrm{QHD}}$ of Eq. (9) are fixed to reproduce the same $m_{N}^{*}$ and $K$ values as listed in Table II for the MQMC model. The coupling constants determined in this way are given in Table III. In the MQMC model, meson-baryon coupling constants are obtained from the quark-meson coupling constants. On the other hand, in QHD mesonnucleon coupling constants provide the starting point for the determination of remaining other meson-baryon coupling constants. Once meson-nucleon coupling constants are fixed from the saturation properties, meson-hyperon coupling constants can be obtained by the quark counting rule (as in Eq. (16)) and the SU(6) symmetry (as in Eq. (17)). The coupling 


\begin{tabular}{|c|c|c|c|c|c|}
\hline$U_{K^{-}}(\mathrm{MeV})$ & -80 & -100 & -120 & -140 & -160 \\
\hline$g_{\sigma K}(\mathrm{MQMC})$ & 1.25 & 2.01 & 2.75 & 3.50 & 4.25 \\
\hline$g_{\sigma K}(\mathrm{QHD})$ & 1.26 & 2.04 & 2.82 & 3.61 & 4.39 \\
\hline
\end{tabular}

TABLE IV: $g_{\sigma K}$ determined for several $U_{K^{-}}$values in the MQMC and QHD models.

constants between strange mesons and hyperons can be obtained by combining the quark counting rule and the $\mathrm{SU}(6)$ symmetry, e.g., $g_{\phi \Lambda}=\sqrt{2} g_{\omega N} / 3$ and $g_{\sigma^{*} \Lambda}=\sqrt{2} g_{\sigma N} / 3$.

\section{Kaon}

There are 5 kaon-meson coupling constants in our models; $g_{\sigma K}, g_{\omega K}, g_{\rho K}, g_{\sigma^{*} K}$ and $g_{\phi K}$. $g_{\omega K}$ and $g_{\rho K}$ can be fixed from the quark counting rule: $g_{\omega K}=g_{\omega}^{q}$ and $g_{\rho K}=g_{\rho}^{q}$ for the MQMC model, and $g_{\omega K}=g_{\omega N} / 3$ and $g_{\rho K}=g_{\rho N}$ for QHD. (Obviously $g_{\rho K}$ from the MQMC model is the same as that from QHD. $g_{\omega K}(=2.71)$ from the MQMC model is essentially the same as $g_{\omega K}(=2.73)$ from QHD.) $g_{\sigma^{*} K}$ may be fixed from $f_{0}(980)$ decay [43], and $g_{\phi K}$ from the $\mathrm{SU}(6)$ relation $\sqrt{2} g_{\phi K}=g_{\pi \pi \rho}=6.04$ [44]. $g_{\sigma^{*} K}$ and $g_{\phi K}$ thus fixed are 2.65 and 4.27, respectively. The remaining coupling constant, $g_{\sigma K}$, can be related to the real part of the optical potential of a kaon at the saturation density through $U_{K^{-}}=-\left(g_{\sigma K} \sigma+g_{\omega K} \omega_{0}\right) . g_{\sigma K}$ values corresponding to several values of $U_{K^{-}}$are listed in Table IV for both MQMC and QHD models.

Thus, out of 5 kaon-meson coupling constants $g_{\rho K}, g_{\sigma^{*} K}$, and $g_{\phi K}$ are the same for both models. $g_{\omega K}$ 's are essentially the same for both models. $g_{\sigma K}$ values are also very similar in both models for all $U_{K^{-}}$values as seen in Table IV. Therefore, all the 5 kaon-meson coupling constants are practically the same for both MQMC and QHD models.

\section{Other quantities relevant to neutron star matter}

To obtain the composition of neutron star matter, we need to determine 16 unknown variables at each matter density, which include 5 meson fields $\left(\sigma, \omega, \rho, \sigma^{*}, \phi\right), 8$ octet baryon densities, 2 lepton densities and the kaon density $\rho_{K}$. Five meson fields can be determined from their equations of motion:

$$
m_{\sigma}^{2} \sigma+\frac{\partial}{\partial \sigma} U_{\sigma}^{\mathrm{QHD}}=\sum_{B} g_{\sigma B} C_{B}(\sigma) \frac{2 J_{B}+1}{2 \pi^{2}} \int_{0}^{k_{B}} \frac{m_{B}^{*}}{\left[k^{2}+m_{B}^{* 2}\right]^{1 / 2}} k^{2} d k+g_{\sigma K} \rho_{K}
$$




$$
\begin{gathered}
m_{\sigma^{*}}^{2} \sigma^{*}=\sum_{B} g_{\sigma^{*} B} C_{B}\left(\sigma^{*}\right) \frac{2 J_{B}+1}{2 \pi^{2}} \int_{0}^{k_{B}} \frac{m_{B}^{*}}{\left[k^{2}+m_{B}^{* 2}\right]^{1 / 2}} k^{2} d k+g_{\sigma^{*} K} \rho_{K} \\
m_{\omega}^{2} \omega_{0}=\sum_{B} g_{\omega B}\left(2 J_{B}+1\right) k_{B}^{3} /\left(6 \pi^{2}\right)-g_{\omega K} \rho_{K} \\
m_{\phi}^{2} \phi_{0}=\sum_{B} g_{\phi B}\left(2 J_{B}+1\right) k_{B}^{3} /\left(6 \pi^{2}\right)+g_{\phi K} \rho_{K} \\
m_{\rho}^{2} \rho_{03}=\sum_{B} g_{\rho B} I_{3 B}\left(2 J_{B}+1\right) k_{B}^{3} /\left(6 \pi^{2}\right)-g_{\rho K} \frac{1}{2} \rho_{K}
\end{gathered}
$$

where $J_{B}$ and $I_{3 B}$ are the spin and the isospin projection, respectively, and $k_{B}$ is the Fermi momentum of the baryon $B$. In Eq. (18) $\frac{\partial}{\partial \sigma} U_{\sigma}^{\mathrm{QHD}}$ term needs to be there only for QHD and is not to be included in the MQMC model. $C_{B}(\sigma)$ and $C_{B}\left(\sigma^{*}\right)$ are determined from the relations $g_{\sigma B} C_{B}(\sigma)=-\frac{\partial m_{B}^{*}}{\partial \sigma}$ and $g_{\sigma^{*} B} C_{B}\left(\sigma^{*}\right)=-\frac{\partial m_{B}^{*}}{\partial \sigma^{*}}$. For $\mathrm{QHD}, C_{B}(\sigma)=C_{B}\left(\sigma^{*}\right)=1$. For MQMC, the explicit forms of $C_{B}(\sigma)$ and $C_{B}\left(\sigma^{*}\right)$ are given in Ref. [18].

Charge neutrality condition of neutron star matter is expressed as

$$
\sum_{B} q_{B} \rho_{B}-\rho_{K}-\rho_{e}-\rho_{\mu}=0
$$

where $q_{B}$ is the charge of baryon $B$ and $\rho_{B}$ is the number density of $B$. Using the charge neutrality and the baryon number conservation conditions, one can fix two quantities, e.g., the density of the neutron and the electron. With these two variables fixed, $\beta$-equilibrium conditions of the baryons give us the following 7 relations for the chemical potentials of $p, \Lambda, \Sigma^{+}, \Sigma^{-}, \Sigma^{0}, \Xi^{-}$and $\Xi^{0}$ as

$$
\begin{gathered}
\mu_{n}=\mu_{\Lambda}=\mu_{\Sigma^{0}}=\mu_{\Xi^{0}} \\
\mu_{n}+\mu_{e}=\mu_{\Sigma^{-}}=\mu_{\Xi^{-}} \\
\mu_{n}-\mu_{e}=\mu_{p}=\mu_{\Sigma^{+}}
\end{gathered}
$$

where the chemical potential of baryon $B$ is given by $\mu_{B}=\sqrt{k_{B}^{2}+m_{B}^{* 2}\left(\sigma, \sigma^{*}\right)}+g_{\omega B} \omega_{0}+$ $g_{\phi B} \phi_{0}+g_{\rho B} I_{3 B} \rho_{03}$. The chemical potential of a non-interacting lepton $l$ is simply written as $\mu_{l}=\sqrt{k_{l}^{2}+m_{l}^{2}}$. The $\beta$-equilibrium condition for leptons

$$
\mu_{e}=\mu_{\mu}
$$



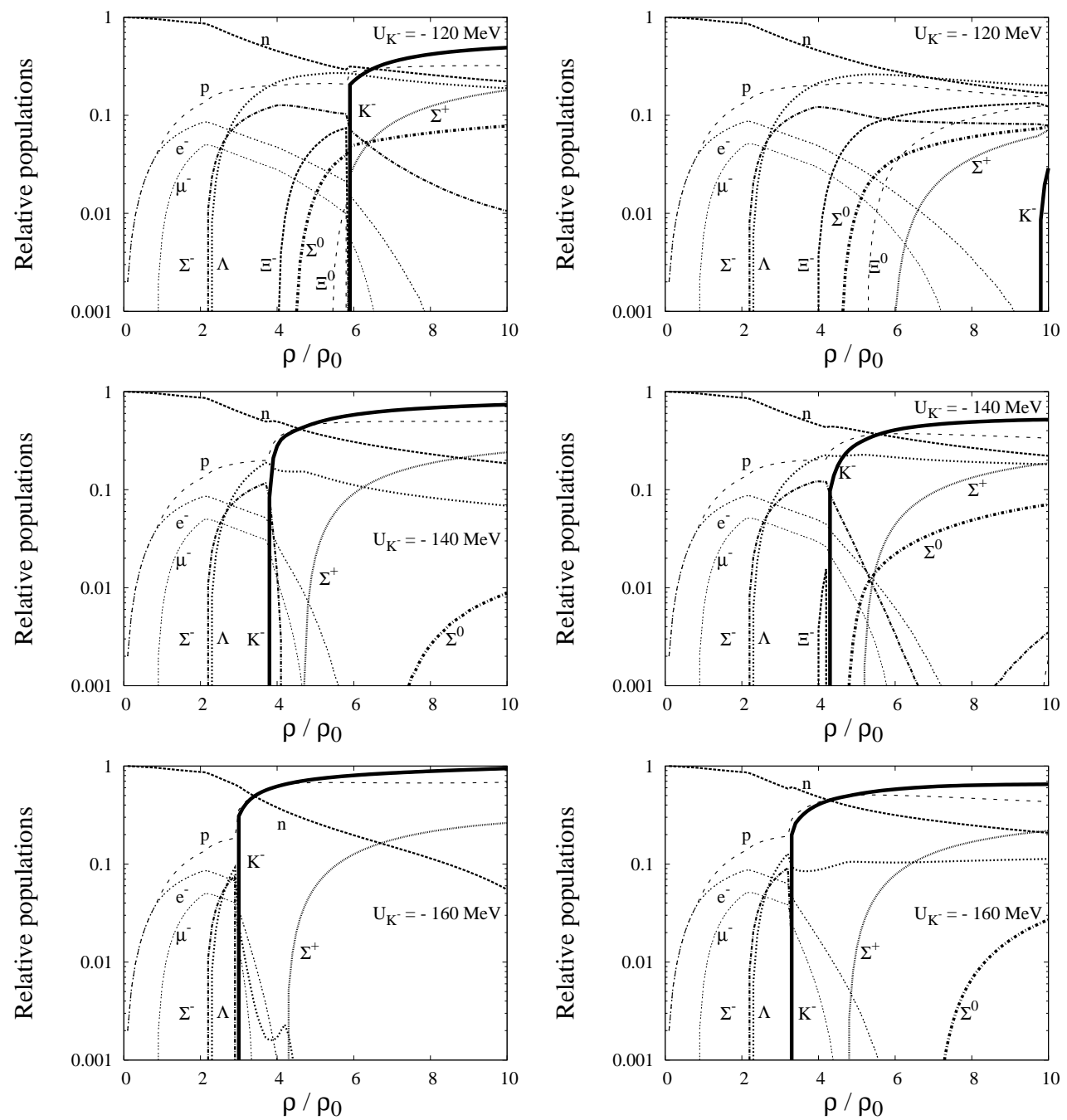

FIG. 1: Compositions of neutron star matter calculated from the MQMC (left panels) and the QHD (right panels) models.

determines the density of muons. At a density where the condition

$$
\omega_{K}=\mu_{n}-\mu_{p}
$$

is satisfied, kaons are condensed and the kaon density $\rho_{K}$ becomes non-zero. Solving the Eqs. (18 26) self-consistently and simultaneously, one can determine the 16 variables uniquely. 


\section{RESULTS}

Fig. 1] shows the relative populations, the ratios of the densities of octet baryons, leptons and $K^{-}$to the total baryon density, in the neutron star matter as functions of $\rho / \rho_{0}$ up to $\rho=10 \rho_{0}$. The left panels show the results from the MQMC model and the right from the QHD model for $U_{K^{-}}=-120,-140$, and $-160 \mathrm{MeV}$. (Figures for $U_{K^{-}}=-80$ and -100 $\mathrm{MeV}$ are not shown here since they are not too much different from the one for $U_{K^{-}}=-120$ $\mathrm{MeV}$ particularly for QHD.) Figures from both models show that the onset density of the kaon condensation $\rho_{\text {crit }}$ becomes lower as $\left|U_{K^{-}}\right|$increases.

To see how $\rho_{\text {crit }}$ changes depending on $U_{K^{-}}$, let us consider Eq. (26), which determines $\rho_{\text {crit }}$. Fig. 2 displays $\omega_{K}$ and $\mu_{n}-\mu_{p}$, which are, respectively, the left and the right hand sides of Eq. (26) (computed without including kaon condensation just for producing this figure). The left panel is from the MQMC model, and the right panel from QHD. At a density where the curve of $\omega_{K}$ intersects with that of $\mu_{n}-\mu_{p}$, kaon condensation sets in. Among the coupling constants and meson fields that determine $\omega_{K}$, only $g_{\sigma K}$ depends on $U_{K^{-}}$. The $\sigma$-meson contributes to $\omega_{K}$ attractively, as can be seen in Eq. (14). Thus $\omega_{K}$ becomes smaller for a larger $\left|U_{K^{-}}\right|$, as shown in Fig. 2 ,

Figure 1 also shows that as $\left|U_{K^{-}}\right|$increases from $120 \mathrm{MeV}$ to $140 \mathrm{MeV} \rho_{\text {crit }}$ changes drastically in both MQMC and QHD models, but as $\left|U_{K^{-}}\right|$increases further above 140 $\mathrm{MeV}, \rho_{\text {crit }}$ changes only moderately. This can also be seen from Fig. 2, As $\left|U_{K^{-}}\right|$increases from $120 \mathrm{MeV}$ to $140 \mathrm{MeV}$ the intersection between the curves for $\mu_{n}-\mu_{p}$ and $\omega_{K}$ moves rapidly (particularly for QHD), whereas when $\left|U_{K^{-}}\right|$increases further above $140 \mathrm{MeV}$ the intersection shifts only a little to lower densities.

Another common feature of the two models is that regardless of $\rho_{\text {crit }}$, once the kaon is created, the density of $K^{-}$piles up very quickly and overwhelms the population of the hyperons and even the nucleons. This behavior was also obtained by other authors [17, 34, 41, 45]. The reason can be partly attributed to the $\omega$-meson. The $\omega$-meson term in the energy of $K^{-}$(in Eq. (14)) has a negative sign and is thus attractive, but it is repulsive for octet baryons. Fig. 3 shows the $\omega$-meson is a dominant meson at higher densities in both MQMC and QHD models. Thus the $\omega$-meson enhances the population of $K^{-}$but suppresses baryons, and the kaon density increases rapidly. In addition, due to the competition between the negatively charged hyperons and $K^{-}$in the charge neutrality condition, the negative 

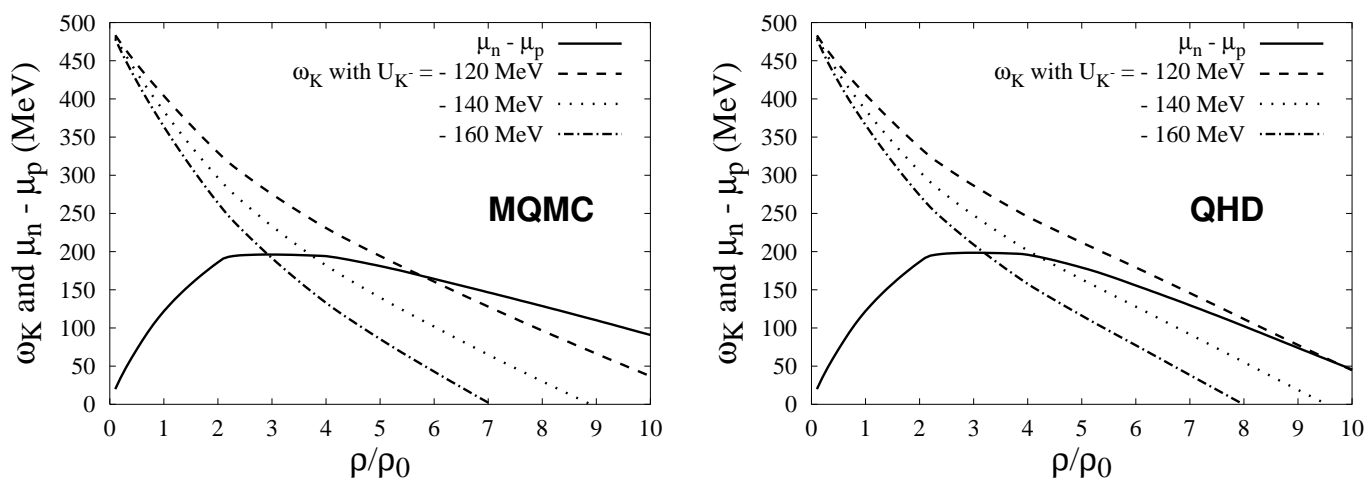

FIG. 2: Kaon energies $\omega_{K}$ for $U_{K^{-}}=-120 \mathrm{MeV}$ (dashed), $-140 \mathrm{MeV}$ (dotted) and $-160 \mathrm{MeV}$ (dot-dashed) are compared with $\mu_{n}-\mu_{p}$ (solid). At the densities where $\omega_{K}$ and $\mu_{n}-\mu_{p}$ intersect, kaons start to condense. The left panel is for the MQMC model, and the right for QHD.
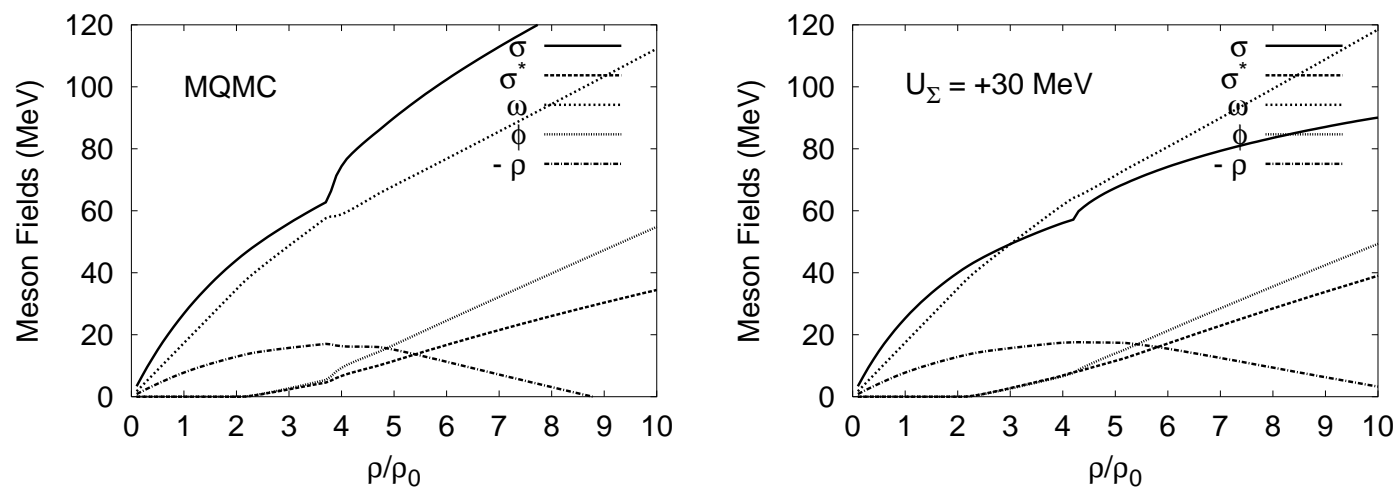

FIG. 3: Meson fields calculated from the MQMC (left) and the QHD (right) models as functions of the matter density for $U_{K^{-}}=-140 \mathrm{MeV}$.

hyperons are highly suppressed and in some cases not even created at all as soon as the kaon condensation sets in. Positively charged hyperons, on the other hand, receive the opposite effects from the kaon condensation, and $\Sigma^{+}$is created at lower densities as $\left|U_{K^{-}}\right|$increases more. The proton density is also enhanced by large abundance of $K^{-}$, which facilitates in turn the enhancement of $\Sigma^{+}$population through the chemical equilibrium condition of the positively charged hyperons in Eq. (24).

Let us now discuss different aspects of the two model calculations. First, Fig. 1 shows that $\rho_{\text {crit }}$ from the MQMC model is lower than that from QHD. For $U_{K^{-}}=-120,-140$, and $-160 \mathrm{MeV}, \rho_{\text {crit }}$ values are $5.9 \rho_{0}, 3.8 \rho_{0}$ and $3.0 \rho_{0}$ in the MQMC model, respectively, 

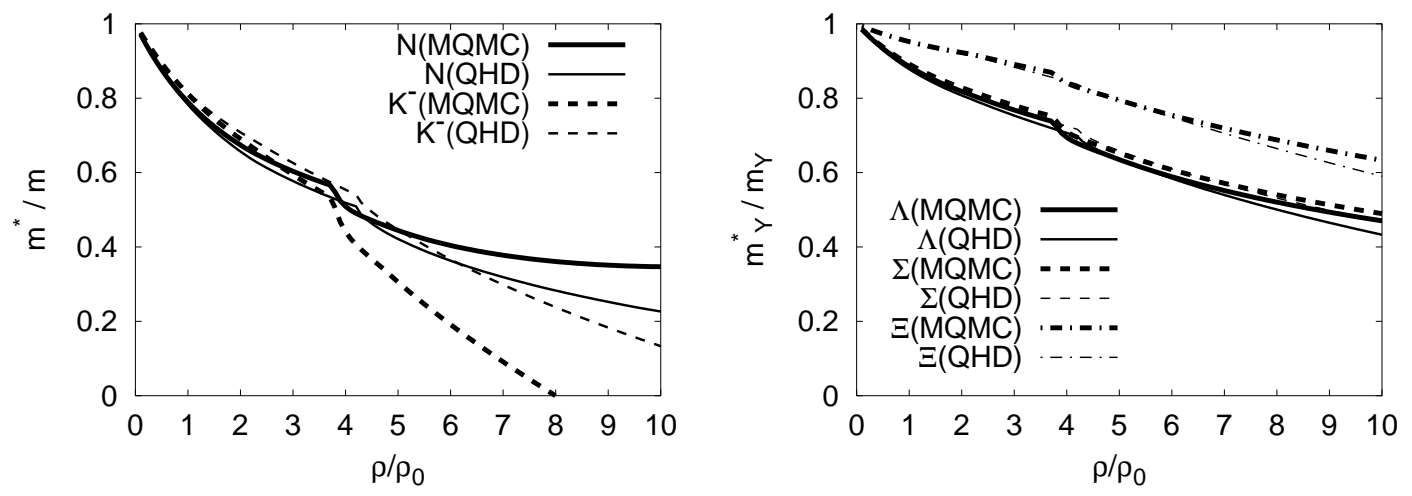

FIG. 4: Effective masses of the nucleons, kaon, and hyperons are plotted for $U_{K^{-}}=-140 \mathrm{MeV}$.

while they are $9.8 \rho_{0}, 4.3 \rho_{0}$ and $3.3 \rho_{0}$ in QHD. Secondly, the MQMC model predicts a larger population of the kaon than QHD for a given $U_{K^{-}}$value. Figure 2 shows that $\omega_{K}$ calculated from the MQMC model decreases more rapidly with density than $\omega_{K}$ from QHD for each $U_{K^{-}}$value. The curves for $\mu_{n}-\mu_{p}$ are more or less the same for both models at $\rho \lesssim 4 \rho_{0}$, but at $\rho>4 \rho_{0} \mu_{n}-\mu_{p}$ decreases faster in QHD. Thus the intersection and kaon condensation occur at lower densities in the MQMC model. This behaviour of the intersection in Fig. 2 is well reflected in the kaon condensation onset density $\rho_{\text {crit }}$ in Fig. 1. Fig. 3 shows that the $\sigma$-meson field calculated by the MQMC model is larger than that calculated by QHD. A larger $\sigma$-field in the MQMC model makes $m_{K}^{*}$ and consequently $\omega_{K}$ smaller. On the other hand, as seen in Fig. 2, $\mu_{n}-\mu_{p}$ from QHD decreases faster with density at higher densities than that from MQMC. Thus the intersection of the $\omega_{K}$ curve with the curve for $\mu_{n}-\mu_{p}$ occurs at lower densities with the MQMC model. Therefore, $\rho_{\text {crit }}$ is smaller in the MQMC model.

Another model dependency of the results can be seen from the population of kaons, which is larger in the MQMC model. The effective mass of a kaon as a point particle is determined by $\sigma$ and $\sigma^{*}$ mesons through the relation $m_{K}^{*}=m_{K}-g_{\sigma K} \sigma-g_{\sigma^{*} K} \sigma^{*}$ and is plotted in Fig. 4. Since the $\sigma$ fields are larger in the MQMC model (as shown in Fig. 3), the effective mass and the energy of a kaon are smaller in the MQMC model than QHD. Thus, kaon condensation takes place more in the MQMC model.

Figures 3 and 4 also show that even though $\sigma$-meson field from the MQMC model is larger than that from QHD as the densities increase, the reduction of the effective mass of baryons is smaller (or similar) with the MQMC model. If one could parametrize the effective 
mass of baryons from the MQMC model in the form of $m_{B}^{*}=m_{B}-g_{\sigma B}(\sigma) \sigma-g_{\sigma^{*} B}\left(\sigma^{*}\right) \sigma^{*}$ where $g_{\sigma B}(\sigma)$ and $g_{\sigma^{*} B}\left(\sigma^{*}\right)$ are functions of $\sigma$ and $\sigma^{*}$, respectively, the results in Fig. 4 might imply that $g_{\sigma B}(\sigma)$ and $g_{\sigma^{*} B}\left(\sigma^{*}\right)$ are decreasing functions with respect to the density. The rate of decrease is rather high since the product $\left(g_{\sigma B}(\sigma) \sigma\right)_{\mathrm{MQMC}}$ is smaller than (or similar to) $\left(g_{\sigma B} \sigma\right)_{\mathrm{QHD}}$ while the $\sigma$-field value from MQMC is much greater than that from QHD. Such a decrease of $g_{\sigma B}(\sigma)$ in the MQMC model may be regarded as partial restoration of the chiral symmetry at high densities.

We have calibrated both the MQMC and QHD model parameters to the same saturation properties. However, we find that the neutron star matter composition profiles from the two models are quite different and that they show significant model dependence. QHD assumes the baryons as point particles, whereas the MQMC model treats the baryons as MIT bags. Thus, the major difference between the two models is in the definition of the effective mass of baryons, $m_{B}^{*}$. The equation of motion for the $\sigma$-meson field is also different accordingly. $m_{B}^{*}$ in QHD is a simple linear function of the $\sigma$-field, and the factor $C_{B}(\sigma)$ in $\mathrm{Eq}(18)$, is a constant. In the MQMC model, $m_{B}^{*}$ is a non-linear function of $\sigma$-field, and thus $C_{B}(\sigma)$ is highly non-linear. When these non-linear $m_{B}^{*}$ and $C_{B}(\sigma)$ are expanded in powers of the $\sigma$-field, an infinite number of $\sigma$-field terms would appear. (Cubic and quartic terms are explicitly taken into account in the QHD model as in Eq. (9).) Higher order terms can be interpreted as higher order contributions such as self interactions of meson fields, which are believed to be more important at high densities. But at high densities it can be questioned whether the non-linear terms of the $\sigma$-meson in the MQMC model account for the higher order effects properly and consistently. For instance, it is generally known that as the baryons come closer to each other the interplay of heavy mesons becomes more important. At high enough densities, their self interaction contributions may need to be included on the same ground as for the $\sigma$-meson, but the present MQMC model truncates the heavy meson terms at the leading order.

It seems worthwhile to discuss at this point two more aspects of our results. The first one is the EoS and the resulting mass-radius relation of the neutron star. The second point is the dependence of our results on the $\Sigma$ hyperon interaction in matter, which is not well known yet.

Let us first consider the EoS and the maximum mass of the neutron star. As the kaon $\left(K^{-}\right)$appears and condensates, the number of negatively-charged hyperons decreases to 


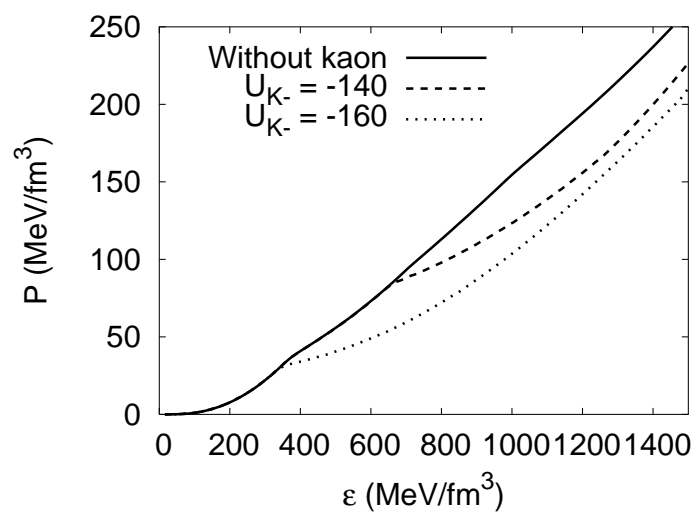

FIG. 5: Comparison of the EoS with and without kaons in QHD model. The Gibbs condition is used to treat the mixed phase.

satisfy the charge neutrality. The decrease in the number of baryons will result in the reduction of the pressure and lead to softnening the EoS. Fig. 5 shows such a softening of the EoS due to the kaon condensation. In calculating the EoS of a system consisting of multicomponent substances, as in the case with the kaon condensation, Gibbs condition has to be employed for the proper description of the mixed phase. The curves in Fig. 5 are the results obtained with the QHD and Gibbs condition. As kaons appear, the EoS becomes considerably soft and the effect becomes more pronounced with a stronger attraction, i.e., for a larger $\left|U_{K^{-}}\right|$value. For the MQMC model, however, applying the Gibbs conditions do not give us a converging solution. Solving the 16 highly nonlinear equations together with Gibbs conditions double the number of equations to be solved, and the convergence could not be reached. It is not clear to us whether the convergence problem is due to numerical problems or due to non-linearity which can cause bifurcation or chaos. Therefore, we have used Maxwell construction for the MQMC model. (Some literatures [46] show that Maxwell construction is a good approximation to the Gibbs condition, but in some other literature [41] it was emphasized that Gibbs condition produces significantly different results from those of Maxwell construction. Below we show that in our case the neutron star mass itself does not change much whether we use Maxwell or Gibbs conditions for QHD. Thus our use of Maxwell construction for the MQMC model may be considered as an acceptable approximation.) We solve Tolman-Oppenheimer-Volkoff equation to calculate the maximum mass of a neutron star. The results are shown in Tab. $\mathrm{V}$, where the central 


\begin{tabular}{c|ccc|ccc|ccc}
\hline & \multicolumn{3}{|c|}{ MQMC $(\mathrm{Mx})$} & \multicolumn{2}{c|}{ QHD $(\mathrm{Mx})$} & \multicolumn{3}{c}{ QHD $(\mathrm{Gb})$} \\
\hline$U_{K^{-}}$ & $\rho_{c} / \rho_{0}$ & $M / M_{\odot}$ & $R$ & $\rho_{c} / \rho_{0}$ & $M / M_{\odot}$ & $R$ & $\rho_{c} / \rho_{0}$ & $M / M_{\odot}$ & $R$ \\
\hline-120 & 6.2 & 1.61 & 11.8 & 6.1 & 1.50 & 11.4 & 6.1 & 1.50 & 11.4 \\
\hline-140 & 4.6 & 1.53 & 12.8 & 5.0 & 1.46 & 12.1 & 5.0 & 1.45 & 12.1 \\
\hline-160 & 4.6 & 1.45 & 13.1 & 4.0 & 1.32 & 12.7 & 4.3 & 1.19 & 12.3 \\
\hline
\end{tabular}

TABLE V: The maximum mass of a neutron star $M$, the corresponding radius $R$ and the density at the center of the star $\rho_{c}$ are listed for $U_{K^{-}}=-120,-140$, and $-160 \mathrm{MeV} . U_{K^{-}}$is in units of $\mathrm{MeV}$, and $R$ in km. "Mx" and "Gb" refer to Maxwell and Gibbs conditions, respectively.
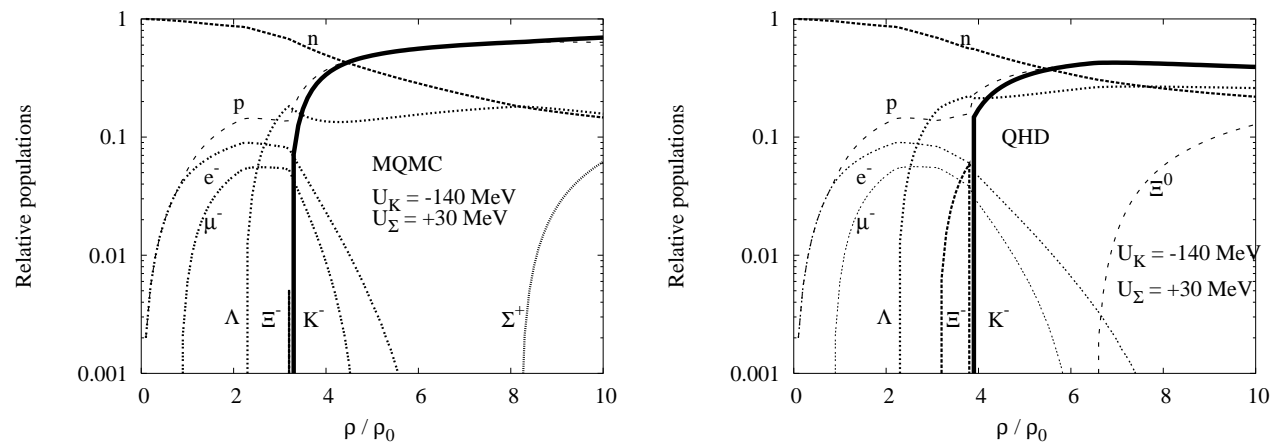

FIG. 6: Comparison of the population from the MQMC (left) with that of the QHD (right). The calculations are done with $U_{\Sigma}=+30 \mathrm{MeV}$ and $U_{K^{-}}=-140 \mathrm{MeV}$.

density, the maximum mass, and the corresponding radius are listed for both MQMC and QHD models. The maximum mass calculated with QHD model is roughly $10 \%$ smaller than that with MQMC model. For both models the maximum mass becomes smaller for a larger $\left|U_{K}\right|$. The maximum mass calculated with MQMC and $\left|U_{K^{-}}\right|=160 \mathrm{MeV}$ is compatible with observation, while the maximum mass calculated with QHD and $\left|U_{K^{-}}\right|=160 \mathrm{MeV}$ becomes too small to be compatible with the observed values. However, this fact may not necessarily rule out the possibility of $\left|U_{K^{-}}\right|$becoming as large as $160 \mathrm{MeV}$ because there are other possible mechanisms which are not included.

Now let us consider the second aspect mentioned above. In the calculations made so far, we have assumed quark-counting rule in determining the hyperon-meson coupling constants. Experiments on $\Lambda$-hypernuclei indicate that quark counting is a good approximation of the 
realistic interaction of $\Lambda$ hyperons in nuclei, which gives the value of $\Lambda$ optical potential at saturation density in the range $-40 \sim-30 \mathrm{MeV}$. On the other hand, there is large ambiguity in the $\Sigma$ hyperon interaction strength. Ref. [47] shows that $\Sigma$ hyperon feels repulsion rather than attraction in nuclear medium. There are also experimental indications that the $\Sigma$ hyperon interaction is repulsive [48]. If the $\Sigma$ interaction is indeed repulsive, the population profile of neutron star matter can change significantly from what is shown earlier in this work since we have used the quark counting rule. The number of $\Sigma^{-}$is closely related to the onset of $K^{-}$condensation since they compete with each other for the charge neutrality condition. To see the effect of possible repulsive nature of $\Sigma$ interaction on the kaon condensation, we have repeated the calculations with repulsive $\Sigma$ interaction. We first fit the coupling constants $g_{\sigma}^{\prime \Sigma}$ in MQMC and $g_{\sigma \Sigma}$ in QHD so that the $\Sigma$ optical potential value at the saturation density equals to $+30 \mathrm{MeV}$, and fix the remaining meson- $\Sigma$ coupling constants with the quark counting rule. The resulting population profiles with the kaon optical potential $U_{K^{-}}=-140 \mathrm{MeV}$ are shown in Fig. 6. Compared to the quark-counting results in Fig. 1, the onset of kaon condensation occurs at slightly lower densities. This minor change happens regardless of $U_{K^{-}}$value. However, the main features of kaon condensation, i.e., its onset density, fast increase of population and its dominance at high densities are not much affected by the change of $\Sigma$ interaction in nuclear medium.

\section{CONCLUSIONS AND DISCUSSIONS}

Using the modified quark-meson coupling model, we have obtained the composition profiles of neutron star matter, focusing on the effects of the strange particles of hyperons and kaons. Motivated by recent theoretical predictions of deeply bound kaonic states [26] and the subsequent claims of the observations of interesting peaks found in KEK [27, 28], FINUDA[30], and BNL[31] experiments, large kaon optical potential $U_{K^{-}}$was considered. By varying the value of $U_{K^{-}}$, we have investigated how the onset density of the kaon condensation and the composition of the stellar matter change. Employing the QHD model parameters which satisfy exactly the same saturation conditions as the MQMC model, we have investigated the model dependence of the results.

We observed two common features from the two model calculations. First, a larger $\left|U_{K^{-}}\right|$produces a smaller onset density of the kaon condensation. This behavior is easily 
understood from the relation between $U_{K^{-}}$and $g_{\sigma K}$ together with the role of $g_{\sigma K}$ to the energy of the kaon $\omega_{K}$. Secondly, the number of kaons rapidly increases, and the number of negatively charged hyperons is strongly suppressed. This is due to the fact that the $\omega$-meson gives rise to attraction to $K^{-}$whereas it couples to baryons repulsively.

Model dependence was also observed. The kaon condensation takes place at lower densities in the MQMC model. The number of kaons is always larger with the MQMC model for given $U_{K^{-}}$values. Larger $\sigma$-meson fields in the MQMC model can explain these behaviors. The differences in the results from the two models become more prominent at larger densities. Growing discrepancies at higher densities have its origin partly in the effective mass of baryons in each model, which greatly affects the self-consistency condition of the $\sigma$-meson. The factor $C_{B}(\sigma)$ in the self-consistency condition of the $\sigma$-meson is highly non-linear in the MQMC model, which can be interpreted as an infinite number of $\sigma$-meson self-interaction terms. These higher order terms may require more proper and consistent treatment at high densities.

An important issue in the dense matter physics is the restoration of the chiral symmetry. According to Ref. [49], not only the mass but also the pion decay constant and meson-nucleon coupling constants decrease at a similar ratio at around the nuclear saturation density. In Ref. [50] the idea of scaling behaviour is applied to the neutron star matter using MQMC and QHD models with only nucleon degrees of freedom, and it is shown that the equation of state becomes stiffer when scaling effects are considered. This implies that if we include a scaling behaviour in our present models, it may ignite the onset of exotic states earlier than the present results which do not include a scaling.

In the kaon sector, the coupling constants of a kaon and exchange mesons are currently an important issue. We took various values of the optical potential of $K^{-}$as an input to fix $g_{\sigma K}$. Other kaon-meson coupling constants are fixed from naive quark counting. It is known, however, that $K^{+}$potential is repulsive with the magnitude $U_{K^{+}} \sim 10 \mathrm{MeV}$ at the saturation density [51]. If $U_{K^{+}}$as well as $U_{K^{-}}$is used as an input, $g_{\sigma K}$ and $g_{\omega K}$ can be determined uniquely. For instance, if we take $U_{K^{-}}=-120 \mathrm{MeV}$ and $U_{K^{+}}=20 \mathrm{MeV}$, then we get $g_{\sigma K}=2.041$ and $g_{\omega K}=4.187 . g_{\sigma K}$ becomes smaller than the value listed in Table IV, while $g_{\omega K}$ becomes nearly twice of $g_{\omega K}$ fixed from the quark counting. Both $\sigma$ and $\omega$ mesons contribute to the $K^{-}$energy attractively, but since the $\omega$ meson becomes a dominant component at higher densities, taking into account of $U_{K^{+}}$can produce appreciably different 
results. It may be interesting to see the effects of $U_{K^{+}}$on the kaon condensation.

In our calculations, we have assumed the kaon as a point particle in both quark and hadron models. Comparison of the two models, however, shows that whether we treat a hadron as a bag (MQMC) or a point particle (QHD) can produce a significant difference. Therefore, it is worthwhile to treat the kaon as a bag and compare the corresponding result with that of a point-like kaon. In Ref. [45] a kaon is treated as a bag in the framework of the QMC model, but no work has been done yet with the MQMC model.

We assume $m_{K}^{*}$ as a linear function of $\sigma$-field, but some authors employ a non-linear form [17, 44]:

$$
m_{K}^{* 2}=m_{K}^{2}-g_{\sigma K} m_{K} \sigma
$$

If we expand this expression in powers of $\sigma / m_{K}$, we obtain

$$
m_{K}^{*} \simeq m_{K}\left[1-\frac{1}{2} g_{\sigma K} \frac{\sigma}{m_{K}}+O\left(\sigma^{2} / m_{K}^{2}\right)\right] \text {. }
$$

The leading order term of the $\sigma$-field has a factor $1 / 2$, which is not present in Eq. (12). Due to the factor $1 / 2$, the rate of decrease in $m_{K}^{*}$ with density would be reduced by a factor 2 , and this would shift the kaon condensation onset density to higher densities. This dependence on the kaon Lagrangian may be worthwhile to be studied.

\section{Acknowledgments}

SWH thanks B. K. Jennings and TRIUMF for hospitality during his sabbatical leave. This work was supported by Korea Research Foundation Grant funded by Korea Government (MOEHRD, Basic Research Promotion Fund) (KRF-2005-206-C00007).

[1] V. R. Pandharipande, Nucl. Phys. A178, 123 (1971).

[2] H. A. Bethe and M. B. Johnson, Nucl. Phys. A230, 1 (1974).

[3] V. R. Pandharipande and R. A. Smith, Nucl. Phys. A237, 507 (1975).

[4] B. Friedman and V. R. Pandharipande, Nucl. Phys. A361, 502 (1981).

[5] J. D. Walecka, Ann. Phys. 83, 491 (1974).

[6] V. R. Pandharipande and R. A. Smith, Phys. Lett. B59, 15 (1975).

[7] S. E. Thorsett and D. Chakrabarty, Astrophys. J. 512, 288 (1999). 
[8] A. R. Villarreal and T. E. Strohmayer, Astrophys. J. 614, L121 (2004).

[9] D. J. Nice, E. M. Splaver, I. H. Stairs, O. Löhmer, A. Jessner, M. Kramer, J. M. Cordes, Astrophys. J. 634, 1242 (2005).

[10] S. I. A. Garpman, N. K. Glendenning and Y. J. Karant, Nucl. Phys. A322, 382 (1979).

[11] A. B. Migdal, Rev. Mod. Phys. 50, 107 (1978).

[12] D. B. Kaplan and A. E. Nelson, Phys. Lett. B175, 57 (1986).

[13] A. R. Bodmer, Phys. Rev. D 4, 1601 (1971).

[14] N. Itoh, Prog. Theor. Phys. 44, 291 (1970).

[15] G. Baym and S. A. Chin, Phys. Lett. B62, 241 (1976).

[16] B. D. Keister and K. S. Kisslinger, Phys. Lett. B64, 117 (1976).

[17] R. Knorren, M. Prakash and P. J. Ellis, Phys. Rev. C 52, 3470 (1995).

[18] S. Pal, M. Hanauske, I. Zakout, H. Stöcker and W. Greiner, Phys. Rev. C 60, 015802 (1999).

[19] N. K. Glendenning, Phys. Rev. D 46, 1274 (1992).

[20] A. Cieply, E. Friedman, A. Gal and J. Mares, Nucl. Phys. A696, 173 (2001).

[21] N. Kaiser, P.B. Siegel and W. Weise, Nucl. Phys. A594, 325 (1995).

[22] J. Schaffner-Bielich, V. Koch and M. Effenberger, Nucl. Phys. A669, 153 (2000).

[23] A. Ramos and E. Oset, Nucl. Phys. A671, 481 (2000).

[24] E. Friedman, A. Gal and C. J. Batty, Nucl. Phys. A579, 578 (1994).

[25] C. J. Batty, E. Friedman, A. Gal, Phys. Rep. 287, 385 (1997).

[26] T. Yamazaki and Y. Akaishi, Phys. Lett. B535, 70 (2002); Y. Akaishi and T. Yamazaki, Phys. Rev. C 65, 044005 (2002).

[27] T. Suzuki, H. Bhang, G. Franklin, K. Gomikawa, R. S. Hayano, T. Hayashi, K. Ishikawa, S. Ishimoto, K. Itahashi, M. Iwasaki, T. Katayama, Y. Kondo, Y. Matsuda, T. Nakamura, S. Okada, H. Outa, B. Quinn, M. Sato, M. Shindo, H. So, P. Strasser, T. Sugimoto, K. Suzuki, S. Suzuki, D. Tomono, A. M. Vinodkumar, E. Widmann, T. Yamazaki, and T. Yoneyama, Phys. Lett. B597, 263 (2004).

[28] T. Suzuki, H. Bhang, G. Franklin, K. Gomikawa, R. S. Hayano, T. Hayashi, K. Ishikawa, S. Ishimoto, K. Itahashi, M. Iwasaki, T. Katayama, Y. Kondo, Y. Matsuda, T. Nakamura, S. Okada, H. Outa, B. Quinn, M. Sato, M. Shindo, H. So, P. Strasser, T. Sugimoto, K. Suzuki, S. Suzuki, D. Tomono, A. M. Vinodkumar, E. Widmann, T. Yamazaki, and T. Yoneyama, Nucl. Phys. A754, 375c (2005). 
[29] M. Iwasaki, Talk at IX International Conference on Hypernuclear and Strange Particle Physics, Oct. 10 - 14, 2006, Mainz, Germany.

[30] M. Agnello, G. Beer, L. Benussi, M. Bertani, S. Bianco, E. Botta, T. Bressani, L. Busso, D. Calvo, P. Camerini, P. Cerello, B. Dalena, F. De Mori, G. D'Erasmo, D. Di Santo, F. L. Fabbri, D. Faso, A. Feliciello, A. Filippi, V. Filippini, E. M. Fiore, H. Fujioka, P. Gianotti, N. Grion, V. Lucherini, S. Marcello, T. Maruta, N. Mirfakhrai, O. Morra, T. Nagae, A. Olin, H. Outa, E. Pace, M. Palomba, A. Pantaleo, A. Panzarasa, V. Paticchio, S. Piano, F. Pompili, R. Rui, G. Simonetti, H. So, S. Tomassini, A. Toyoda, R. Wheadon, and A. Zenoni Phys. Rev. Lett. 94, 212303 (2005).

[31] T. Kishimoto, T. Hayakawa, S. Ajimura, S. Minami, A. Sakaguchi, Y. Shimizu, R. E. Chrien, M. May, P. Pile, A. Rusek, R. Sutter, H. Noumi, H. Tamura, M. Ukai, Y. Miura, and K. Tanida, Nucl. Phys. A754, 383c (2005).

[32] J. Mares, E. Friedman, A. Gal, Nucl. Phys. A770, 84 (2006).

[33] X. Jin and B. K. Jennings, Phys. Lett. B374, 13 (1996); Phys. Rev. C 54, 1427 (1996).

[34] S. Banik and D. Bandyopadhyay, Phys. Rev. C 64, 055805 (2001).

[35] B. D. Serot and J. D. Walecka, Adv. Nucl. Phys. 16, 1 (1986).

[36] S. Fleck, W. Bentz, K. Shimizu and K. Yazaki, Nucl. Phys. A510, 731 (1990).

[37] K. Saito and A. W. Thomas, Phys. Lett. B327, 9 (1994).

[38] K. Saito, K. Tsushima, and A. W. Thomas, hep-ph/0506314.

[39] C. Y. Ryu, C. H. Hyun, J. Y. Lee, and S. W. Hong, Phys. Rev. C 72, 045206 (2005)

[40] J. Boguta and A. R. Bodmer, Nucl. Phys. A292, 413 (1977).

[41] N. K. Glendenning and J. Schaffner-Bielich, Phys. Rev. C 60, 025803 (1999).

[42] I. Zakout, W. Greiner and H. R. Jaqaman, Nucl. Phys. A759, 201 (2005).

[43] T. A. Armstrong et al., WA76 Collaboration, Z. Phys. A 51, 351 (1991).

[44] J. Schaffner and I. N. Mishustin, Phys. Rev. C 53, 1416 (1996).

[45] D. P. Menezes, P. K. Panda and C. Providencia, Phys. Rev. C 72, 035802 (2005).

[46] T. Maruyama, T. Tatsumi, D. N. Voskresensky, T. Tanigawa, T. Endo, S. Chiba, Phys. Rev. C 73, 035802 (2006); T. Endo, T. Maruyama, S. Chiba, T. Tatsumi, Prog. Theor. Phys. 115, 337 (2006).

[47] J. Mares, E. Friedman, A. Gal and B. K. Jennings, Nucl. Phys. A594, 311 (1995).

[48] P. K. Saha, H. Noumi, D. Abe, S. Ajimura, K. Aoki, H. C. Bhang, K. Dobashi, T. Endo, 
Y. Fujii, T. Fukuda, H. C. Guo, O. Hashimoto, H. Hotchi, K. Imai, E. H. Kim, J. H. Kim, T. Kishimoto, A. Krutenkova, K. Maeda, T. Nagae, M. Nakamura, H. Outa, T. Saito, A. Sakaguchi, Y. Sato, R. Sawafta, M. Sekimoto, Y. Shimizu, T. Takahashi, H. Tamura, L. Tang, K. Tanida, T. Watanabe, H. H. Xia, S. H. Zhou, X. F. Zhu, and L. H. Zhu, Phys. Rev. C 70, 044613 (2004).

[49] G. E. Brown and M. Rho, Phys. Rev. Lett. 66, 2720 (1991).

[50] C. H. Hyun, M. H. Kim, and S. W. Hong, Nucl. Phys. A718, 709c (2003); C. Y. Ryu, C. H. Hyun, S. W. Hong and B. K. Jennings, Eur. Phys. J. A 24, 149 (2005).

[51] G. Q. Li, C.-H. Lee and G. E. Brown, Phys. Rev. Lett. 79, 5214 (1997). 